\title{
A!
}

This is an electronic reprint of the original article.

This reprint may differ from the original in pagination and typographic detail.

Diz, Gustavo Souto dos Santos; Hamacher, Silvio; Oliveira, Fabricio

\section{A robust optimization model for the maritime inventory routing problem}

Published in:

Flexible Services and Manufacturing Journal

DOI:

10.1007/s10696-018-9327-9

Published: 17/09/2018

Document Version

Peer reviewed version

Please cite the original version:

Diz, G. S. D. S., Hamacher, S., \& Oliveira, F. (2018). A robust optimization model for the maritime inventory routing problem. Flexible Services and Manufacturing Journal, 31, 675-701. https://doi.org/10.1007/s10696-0189327-9

This material is protected by copyright and other intellectual property rights, and duplication or sale of all or part of any of the repository collections is not permitted, except that material may be duplicated by you for your research use or educational purposes in electronic or print form. You must obtain permission for any other use. Electronic or print copies may not be offered, whether for sale or otherwise to anyone who is not an authorised user. 
Title: A robust optimization model for the maritime inventory routing problem

\author{
Authors: \\ Gustavo Souto dos Santos Diz \\ gustavodiz@petrobras.com.br \\ Phone number: (55) 21993114650 \\ Logistic Department, Petrobras \\ Av. Henrique Valadares, $28-11^{\circ}$ andar - Torre B \\ 20231-030, Rio de Janeiro, RJ, Brazil \\ Silvio Hamacher \\ hamacher@puc-rio.br \\ Industrial Engineering Department, PUC-Rio \\ Rua Marques de São Vicente, 225, Rio de Janeiro, RJ, Brazil \\ Fabricio Oliveira \\ fabricio.oliveira@aalto.fi \\ System Analysis Laboratory, Department of Mathematics and Systems Analysis, \\ School of Science, Aalto University \\ FI-00076 AALTO, Finland
}




\section{A robust optimization model for the maritime inventory routing problem}

\section{Abstract}

Uncertainty is a highly important aspect of maritime transportation. Unforeseen occurrences related to environmental conditions, poor weather, vessel reliability, or port congestion are frequent and have a non-negligible impact on the total time required for vessels to perform (un)loading operations at ports. We study a special case of maritime transportation named the maritime inventory routing (MIR) problem, in which one must determine the routings of vessels while keeping the inventory levels at ports within the operational limits. In this paper, we propose a robust optimization approach that considers the uncertainty in the total time spent by vessels at the ports. This approach allows the trade-off between the risk of infeasibility (i.e., violating inventory limits at ports) and the increase in operational costs due to the protection against uncertainty events to be assessed. To test the proposed methodology, we used a real-world instance based on the MIR problem faced by a Brazilian petroleum company. In this problem, violating the inventory limits at ports causes considerable financial losses due to consequent interruptions in crude oil production. Our approach supports the decision maker to devise more robust plans in which the risk of violating inventory limits is acceptable. In other words, despite the increase in the operational costs associated with more robust solutions, the approach enables the decision maker to avoid much larger potential costs. For the problem considered, we observed that the probability of infeasibility of the proposed solution may be reduced from $87 \%$ to $2 \%$, depending on the level of robustness adopted by the decision maker. However, this increased protection causes an increase of up to $13 \%$ in the overall costs.

Keywords: maritime inventory routing, mathematical programming, robust optimization, uncertainty 


\section{Introduction}

Uncertainty is a highly important aspect of maritime transportation. In real-life problems, unforeseen occurrences related to environmental conditions, poor weather, vessel reliability or port congestion are very frequent and have direct impacts on vessels' operations (Agra et al., 2015). These events may cause delays or interruptions in some operations, thus making it difficult to predict how long the operations will last.

In this paper, we study a maritime transportation problem known as maritime inventory routing (MIR), and we use the case of a Brazilian petroleum company to illustrate the proposed framework. In the MIR problem, the player responsible for carrying the crude oil from production ports to consumer ports is also in charge of managing crude oil inventory levels at these ports. Real-life MIR problems, especially in the petroleum industry, are highly capital-intensive (Diz et al. 2017). Therefore, a small improvement in the coordination of planning inventory management and ship-scheduling activities may result in great economic benefits (Christiansen et al., 2004; Christiansen et al., 2013; Agra et al., 2013). In addition to operational costs, the costs of violating inventory limits may be prohibitive in real-life MIR problems. For example, in the petroleum industry, disrupting an inventory bound may represent a loss of crude oil production or a reduction in the refining processing level, both of which would cause a significant loss of income. If we consider a crude oil barrel price of USD 50.00 and a crude oil production of 60,000 barrels a day (i.e., an offshore medium size facility), an interruption in the production process would mean USD 3.0 million in losses per day.

However, despite the great influence of uncertainty over real-life MIR problems, most previous studies present deterministic approaches to solve them (Christiansen et al. 2013, Papageorgiou et al. 2014, Agra et al. 2015). However, uncertain events are responsible for a large fraction of the operational costs. To protect against uncertainty, the player responsible for transportation usually oversizes its fleet, aiming to ensure that the crude oil production will be streamlined and no interruptions will occur due to a lack of offloading vessels. Uncertainty increases the maritime operational costs, compromises the efficiency of the fleet, and makes ship-scheduling decisions even more difficult, thus raising the risk of violating inventory limits. 
The purpose of this paper is to present a robust optimization approach to solve MIR problems by considering the occurrences of unforeseen events that impact ship scheduling decisions. Robust optimization provides solutions that are robust enough to remain feasible, even in the worst-case realization of uncertain parameters (Soyster, 1973). Bertsimas and Sim (2004) proposed a development on Soyster's approach in order to regulate the level of robustness of the solution and reduce the excessive level of conservativeness of the solution. We present these approaches in Section 3. We also demonstrate how this robust approach can leverage fleet efficiency, reduce the risks of violating inventory limits, and control maritime operational costs.

The contributions of this paper are twofold. The first contribution is to provide a framework that can be used to support the decision maker to quantify the risks of violating inventory bounds at ports (infeasible solutions). We strongly believe that this is of great value to solve real-life MIR problems where the costs of violating inventory limits are much larger than transportation costs. The second contribution is the assessment of how the level of conservativeness chosen by the decision maker influences total operational costs while also evaluating the trade-off between operational costs and the risks of infeasibility. To the best of our knowledge, this is the first time in the literature that such a trade-off assessment has been presented in the context of MIR problems.

The remainder of this paper is organized as follows. In Section 2, we present the MIR problem faced by a Brazilian petroleum company. In Section 3, we describe a brief review of the literature about MIR problems and the robust optimization approach that considers uncertainty in the time spent by vessels at ports. In Section 4, we discuss the computational experiments and the results of the robust optimization approach implemented in a practical case. Finally, in Section 5, we present the main conclusions of this study and future research directions.

\section{Problem description}

In the MIR problem considered in this paper, most of the crude oil production occurs offshore with the use of vessels called Floating Production Storage and Offloading (FPSO). This makes the company highly dependent on maritime transportation to offload its production. The company is responsible for every step of its supply chain, including the oil and gas exploration, production, transportation, storage and refinement, and distribution of refined products. 
Next, we describe first the deterministic problem, then the sources of uncertainties and why they are relevant in the planning process.

\subsection{Real-life MIR problem}

Our MIR problem consists of managing crude oil inventory levels at the FPSOs, transporting crude oil using a heterogeneous fleet of vessels from the FPSOs to the onshore terminals, and ensuring that inventory levels at these terminals will not fall under a minimum limit. Figure 1 represents the flow of crude oil in the company's supply chain. The ship scheduling activity defines on a daily basis which ships will carry crude oil from the FPSOs to the refineries. Usually, the schedulers decide the voyages for the next day as well as the offloading operations that must occur approximately five days ahead. Every day, the schedule is updated with those vessels that will be available for a new voyage on the next day. Voyages from FPSOs to onshore terminals take from 1 to 3 days of sailing time. Meanwhile, the time spent at the FPSOs and at the terminals to load and discharge operations ranges from 1 to 6 days or longer, depending on several uncertain factors.

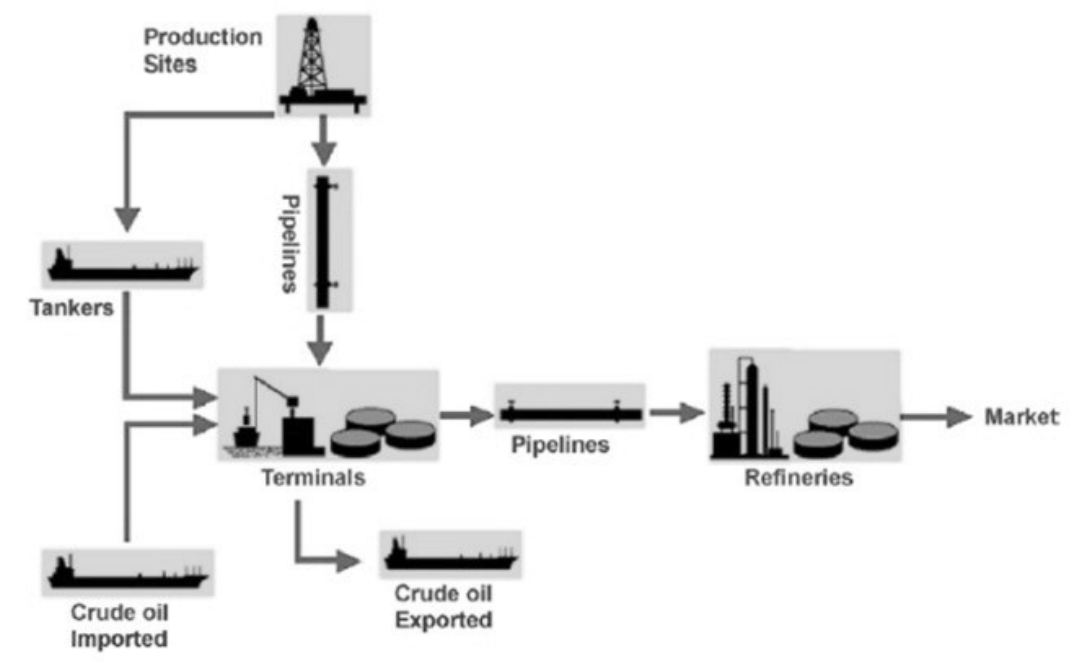

Figure 1: The flow of crude oil in the company's supply chain.

Prior to scheduling the fleet, a planning team combines the vessels into groups and assigns each of these groups to a geographic region (referred to as subsystems). Figure 2 represents each subsystem and the location of FPSOs and onshore terminals. In the case under study, each group of vessels is pre-allocated to a subsystem (forming somewhat independent MIR problems) in which they will transport crude oil from the FPSOs to the onshore terminals. These vessels can be reallocated according to the transportation demand of each subsystem. However, due to the physical and operational characteristics 
of the fleet, onshore terminals and production sites may restrict interchanging vessels between them.

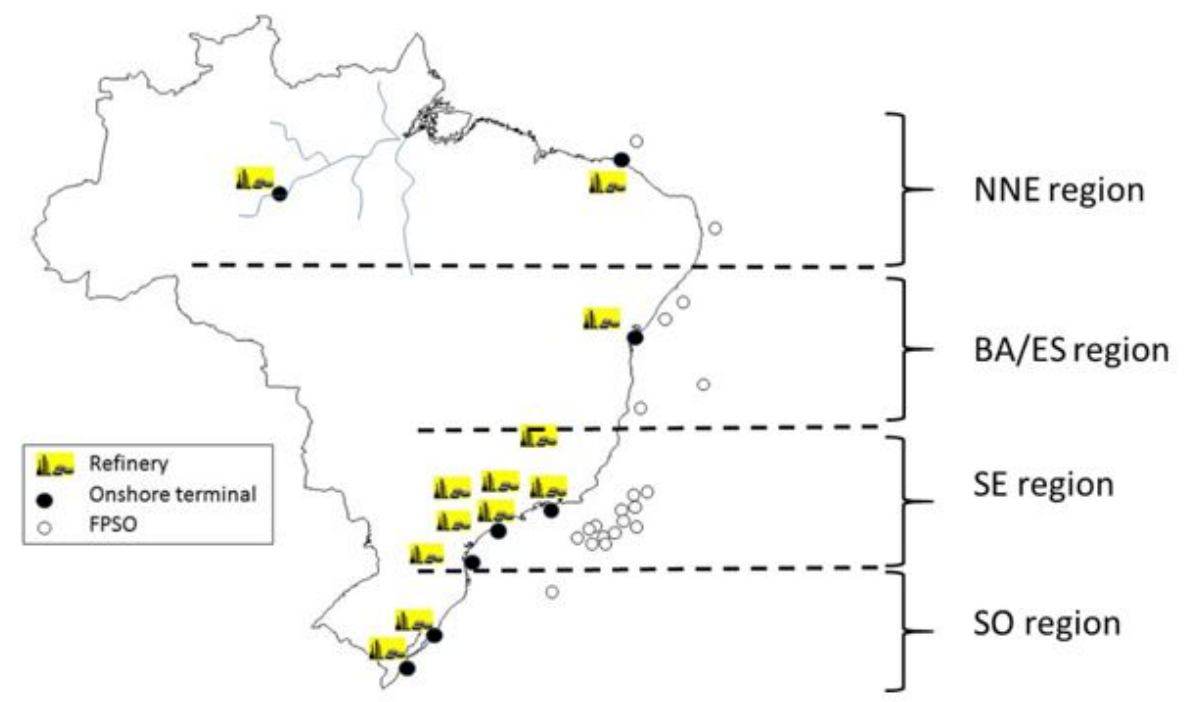

Figure 2: The fleet is divided into regions and assigned to each of the subsystems.

The fleet is heterogeneous in terms of tank capacity, costs, speed and bunker consumption. Bunker consumption represents most of the variable transportation costs, while the other cost component is associated with port fees. The fixed part of maritime transportation costs are the hiring costs, known in the shipping industry as the hire fee.

In what follows, we describe in more details some of the main uncertain aspects involved in the real-life MIR problem being considered.

\subsection{Uncertainty aspects}

In maritime transportation, uncertain events are very frequent and are often related to environmental conditions. However, poor weather, wind, and waves are just some of the conditions that may cause operations to be delayed, interrupted, or even aborted.

In MIR problems, most of the uncertain events will have some impact on the time spent by the vessel on some activity (sailing, loading or discharging). In the case considered here, the time spent by the fleet on sailing is approximately $25 \%$ of its total available time. Most voyages are short, and hence speed variations have little impact on the total sailing time. However, the time spent by the vessels at ports ${ }^{1}$ (FPSO or at onshore terminals) represents the remaining $75 \%$ of available time. During the time spent at ports, a vessel

\footnotetext{
${ }^{1}$ We refer to FPSOs as "ports" because the vessel must moor to the FPSO for the unloading to be performed.
} 
may be waiting for berthing, performing berthing or unberthing operations, loading or discharging, or even waiting for the next voyage. Since the time spent by vessels at ports represents a much larger fraction of the available time of the fleet, we focus our attention on the uncertain events that impact this fraction of the time.

Operational delays may occur due to a diverse range of causes. At a production site, it may occur due to bad weather, vessel or FPSO unreliability, and a lack of crude inventory, for example. At onshore terminals where the vessels discharge the crude oil, bad weather, equipment degradation, berth congestion, the lack of tank capacity, bureaucracy reasons (vessel clearance) and pilot delays are some examples of unforeseen occurrences that cause delays. After berthing procedures, the operational time is usually increased by many sorts of events, such as vessel's or terminal's pump unreliability, vessel clearance, the lack of tank capacity, bad weather (that might interrupt or delay a pumping operation), and numerous additional unexpected reasons.

Therefore, unforeseen occurrences directly influence the time spent by the vessels in their operations, thus causing delays in sailing, discharging and/or loading operations. They provoke some undesirable effects such as the inefficient use of the fleet, higher transportation costs, and a higher risk of violating inventory limits.

\section{MIR problem under uncertainty}

Christiansen (1999) and Flatberg et al. (2000) were the pioneer studies concerning MIR problems. Since then, researchers have struggled to overcome the challenges posed by MIR problems (Chistiansen and Fagerholt, 2009; Christiansen et al., 2011). According to Furman et al. (2011), Agra et al. (2013) and Papageorgiou et al. (2014), MIR problems are very challenging to solve to optimality in real-life instances. Usually, this type of problem has weak linear relaxation bounds, which makes it harder for commercial solvers (that rely on the branch-and-bound algorithm) to find optimal solutions. In the face of such computational complexity, Song and Furman (2013) claimed that their model and algorithm framework is flexible and effective enough to be a choice model and solution method for practical inventory routing problems. Rocha et al. (2013) proposed valid inequalities named Cascading Knapsack inequalities to improve computational performance. To tighten the linear relaxation bounds, Agra et al. (2013) proposed a reformulation of a discrete time MIR model that they named the Fixed Charge Network Flow (FCNF) formulation and implemented important valid inequalities that strengthened 
the method. Papageorgiou et al. (2014) proposed a general formulation that incorporates assumptions and families of constraints that are most prevalent in practice. Papageorgiou et al. (2014) also presented a review of MIR problems and the first library of MIR problem instances in the literature.

Few studies in the literature feature uncertainty in MIR problems. Christiansen and Nygreen (2005), Christiansen et al. (2007) and Papageourgious et al. (2014) proposed setting an upper safety stock level that is below the storage capacity, and a lower safety stock level that is above a specified lower inventory level. Any diversion of the inventory from this range of safety stock limits was then penalized. Most recently, some authors adopted optimization under uncertainty techniques to address uncertainty in production/consumption rates or in travel time through the adoption of inventory safety levels in inventory planning (Halvorsen-Weare et al., 2013; Agra et al., 2015; Zhang et al. 2015; Assis and Campnogara, 2016; Agra et al., 2016; Zhang et al., 2018; Agra et al., 2018).

To address the uncertain aspects of sailing times and production levels, Halvorsen-Weare et al. (2013) proposed a solution method that adopted several robust strategies and a simulation model to evaluate solutions. Agra et al. (2015) presented a two-stage stochastic programming model with recourse for a stochastic MIR problem with uncertain sailing times and unpredictable waiting times at ports. Zhang et al. (2015) studied a general robust maritime inventory routing problem with time windows, where the length and placement of the time windows in the planning horizon are also decision variables. They formulate the problem as a two-stage stochastic mixed-integer program and consider stochastic travel times. Assis and Campnogara (2016) presented a MILP model and a combination of a MILP formulation and heuristics that included relax-and-fix and rollinghorizon. Agra et al. (2016) proposed a two-stage stochastic programming model with recourse to deal with uncertain sailing times. Zhang et al. (2018) formulated the problem as a two-stage stochastic mixed-integer program and proposed a two-phase solution approach that considers disruptions in the planned delivery routes. Agra et al. (2018) investigated the use of adaptive robust optimization, where the sailing times are assumed to belong to the well-known budget polytope uncertainty set. In their recourse model, the routing, the order of port visits, and the quantities to load and unload are fixed before the uncertainty is revealed, while the visit time to ports and the stock levels can be adjusted after the uncertainty is observed. 


\subsection{Robust optimization approach}

In the MIR problem at hand, we consider uncertainty in the time spent by vessels at ports due to delays in the vessels operations and the amount of time spent by vessels at ports (approximately $75 \%$ of the total fleet time). As the costs of infeasibility (i.e., violating inventory limits at ports) are much higher than operational costs, we decided to use a robust optimization technique that emphasizes feasibility over optimality.

Soyster (1973) first proposed the robust optimization approach, but the solutions provided by its approach were too conservative. Gabrel et al. (2014) claimed that the complete protection from adverse realizations often comes at the expense of a severe deterioration in the objective function. According to Gorissen et al. (2015), robust optimization (RO) is a relatively young and active research field, and it has mainly developed over the last 15 years. Ben-Tal and Nemirovisk (2000) and Bertsimas and Sim (2004) proposed less conservative models than Soyster (1973), in which one could regulate the level of robustness to be adopted. They assume that very rarely will all of the uncertain parameters occur in their worst possibility. Therefore, they could accept less robust solutions. However, unlike Ben-Tal and Nemirovski (2000), the robust counterparts proposed by Bertsimas and Sim (2004) are linear optimization problems, and thus their approach is readily generalized to discrete optimization problems.

The approach proposed in Bertsimas and Sim (2004) deterministically protects the model against violations of constraints $i$ by using a coefficient of uncertainty $\Gamma_{i}$ that is used to adjust the solution robustness against the decision-maker conservatism. In other words, it ensures that the solution is feasible up to $\Gamma_{i}$ uncertain coefficient changes. This term is often referred to as the budget of uncertainty and reflects the decision-maker's attitude towards uncertainty. As this budget increases, the model is more protected against deviations in uncertain parameters. In the MIR problem robust optimization model we study here, the coefficient of uncertainty $\Gamma_{i}$ represents the number of visits in each port, where the time spent by vessels at port may deviate from the nominal time to the maximum allowed time spent at port. Therefore, as we increase the level of robustness $\left(\Gamma_{i}\right)$, the time spent at port may deviate in more visits. For example, if $\Gamma_{i}=0$, then no visit in port $i$ may deviate from nominal time and if $\Gamma_{i}=5$, then the time spent by vessels at port may deviate in 5 visits at port $i$. Bertsimas's and Sim's (2004) approach allows the decision maker to regulate the level of robustness required from a given solution, thus avoiding over-conservative solutions. 
We based our robust approach on the Bertsimas and Sim (2004) technique. In the next section, we present the MIR problem formulation and the robust optimization approach applied to MIR problems.

\subsection{Robust optimization approach for the MIR problem}

In the robust optimization based approach for the MIR problem (RO-MIR), the aim is to minimize the transportation costs while finding a feasible solution that maintains the inventory levels between operational limits. However, in the RO-MIR, when delays in time spent at ports are observed, the proposed ship scheduling consumes more days of the fleet vessels. Thus, one way of protecting the solution against those delays is "consuming" more vessels, which means using vessels for a longer period of time for the same voyage. Therefore, we consider it important to take into account the fixed costs (hiring costs) in the RO-MIR problem. In this sense, we included the fixed costs of the fleet in the problem in order to measure the impacts of the uncertainty in the fleet size and, consequently, in the transportation costs.

The need for more or fewer vessels to transport all the crude oil of a subsystem does not mean that the ship scheduling team will charter a new vessel or redeliver a vessel of the fleet. They are allowed to reallocate one vessel to a different geographic subsystem, if necessary (see Section 2.1). They are responsible for the fleet sizing decisions in the shortterm and for maintaining the service level (i.e., keeping inventory levels at FPSOs and onshore terminals within their operational range). While the scheduling decisions that result from the robust methodology will be important to ascertain the feasibility of a given plan, the most important decision concerns the number of vessels dedicated to each subsystem for the next short-term planning horizon.

The RO-MIR model is based on the formulation first proposed by Agra et al. (2013). They reformulated a discrete time model as the Fixed Charge Network Flow (FCNF) model that they claim provides tighter linear relaxations when compared with the other discrete time models found in the literature. Diz et al. (2017) successfully applied the same formulation to a subsystem of a MIR problem faced by a Brazilian petroleum company. A list of symbols used in this paper is presented in Appendix 1. The basic FCNF formulation proposed by Agra et al. (2013) is presented in Appendix 2.

In the next section, we describe the robust optimization approach. 


\subsection{Applying the robust optimization framework}

To explicitly quantify the relationship between the level of robustness of the solution and the probability of a constraint violation, we associate the Bertsimas and Sim (2004) approach with a Monte Carlo simulation process. By simulating the uncertain time spent by vessels at the port, one is able to estimate the probability of infeasibility for the solution of each level of robustness that is provided by the robust optimization model.

Our robust optimization approach has three phases. First, we reformulate FCNF model and apply Bertsimas and Sim (2004) approach in order to obtain the robust optimization fixed charge network flow (RO-FCNF). In the second phase, we execute the RO-FCNF model for each level of robustness (using a matheuristic based on relax-and-fix and fixand-optimize to reduce computational time) and vary the budget of uncertainty $\Gamma$ (level of robustness). In the third phase, we execute a Monte Carlo simulation to estimate the probability of infeasibility for each level of $\Gamma$. Figure 3 illustrates the methodology that we adopted.

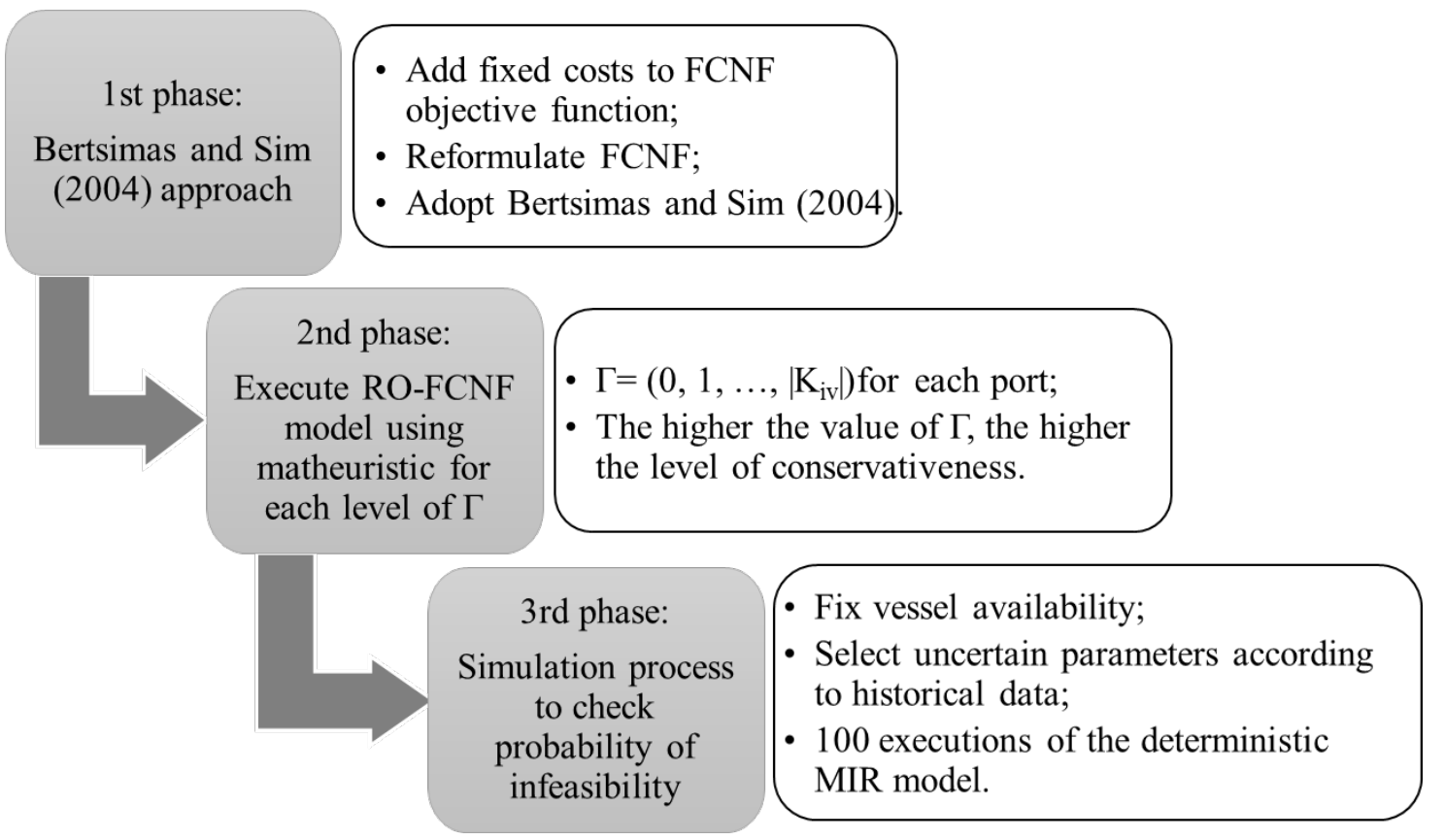

Figure 3: Methodology flowchart of the proposed approach.

Next, we describe each phase of the methodology proposed.

\subsubsection{First phase}

In the first phase of methodology, we reformulate the FCNF model to add the fixed costs of the fleet and to consider the nominal time spent by vessels at ports. Such reformulations 
allow the uncertainty in the time spent at ports to be modeled. We present in Appendix 2 the reformulations proposed here.

To formulate the RO-FCNF model, we apply the robust optimization approach proposed in Bertsimas and Sim (2004) to the modified FCNF model. Under robust optimization approach, we are willing to accept a suboptimal solution for the nominal values of time spent by vessels at each port in order to ensure that the solution remains feasible and near optimal when the data changes. The approach of Bertsimas and Sim (2004) attempts to make such trade-off more attractive; that is, such approach flexibly adjusts the level of conservativeness of the robust solutions in terms of probabilistic bounds of constraint violation.

The development and implementation of approach proposed by Bertsimas and Sim (2004) to the modified FCNF model are described in detail in Appendix 3.

\subsubsection{Second phase}

In the second phase, we execute the RO-FCNF model for each level of robustness and vary the budget of uncertainty $\Gamma$ from 0 to a pre-defined maximum value of $\Gamma$. For each level of $\Gamma$, we obtain a robust solution that consists of a solution that will remain feasible even if the realization of the uncertain parameter occurs in its worst case in every visit of vessel $v$ to port $i$ up to $\Gamma$ occurrences ( $\Gamma$ defines the maximum number deviations from the nominal time). As the MIR problem is a computationally complex model, we introduce a matheuristic based on the combination of the relax-and-fix and fix-andoptimize methods to improve quality of solutions and reduce the computational time during the second phase. Diz (2017) used these matheuristics in a MIR problem under uncertainty and claims that they reduced significantly computational time and slightly improved the objective function value when compared to directly solving the MIP using commercial software.

The heuristic approach proposed by Diz (2017) combined the relax-and-fix algorithm to find an initial solution for the original problem, and the fix-and-optimize heuristic to improve such initial solution. Both heuristics are called matheuristics because they are based on an iteration process, where on each iteration some integer variables of original problem are fixed and/or relaxed and a decomposed subproblem is then solved using a commercial MIP solver. Such iteration process is repeated aiming to improve an initial solution until a stop condition is reached. 
The process of choosing the variables to be fixed or relaxed during the matheuristic iteration may be aleatory or not. In our case, we decided not to use a random process, but to use instead an index of the model to choose the variables to be fixed or relaxed at each iteration of the algorithm. For example, when using the index ports, we order the ports $i \in N$ arbitrarily and select a subset of ports (say $N^{\prime} \subset N$ ) such that all variable indexed by those ports $i \in N^{\prime}$ will be fixed (or relaxed) at a given iteration. Such subset $N^{\prime}$ of ports is modified (by successively removing ports from $N^{\prime}$ and including ports from $N \backslash N^{\prime}$ ) from one iteration to another until the algorithm terminates. Diz (2017) applied relaxand fix and fix and optimize to a MIR problem. The author demonstrated that using the index ports to fix the integer variables provided better initial solutions than when using index time, for example. When it comes to the fixand-optimize heuristic, the use of index vessels improved the initial solution more frequently than when the index time was used. Comprehensive details concerning the implementation of the aforementioned heuristics and computational tests comparing the performance for different configurations and selection of index sets to be fixed or relaxed are provided in Diz (2017).

\subsubsection{Third phase}

The third phase of the framework consists of assessing the robust solutions in terms of their probability of infeasibility for each level of robustness. For each level of $\Gamma$, we obtain a distinct ship routing and scheduling for the fleet. However, ship scheduling decisions are heavily influenced by day-by-day uncertain events and may be adjusted with short notice as these uncertain events unfold. The important information we carry from the optimization model to the simulation phase is the number of vessels utilized and their respective availabilities, obtained for each level of $\Gamma$. The vessel availability is given by the time between the time period in which it leaves the artificial origin node $O_{v}$ and the time period it enters the artificial destination node $D E_{v}$. We name such vessel availability the protection provided by the RO-FCNF for each level of robustness against infeasibility. We demonstrate how to model the vessel availability on the deterministic FCNF model in Appendix 4.

Once we define the protection of each level of robustness provided in the second phase, we evaluate the chances of a robust solution being infeasible. To do so, for each robust solution, we execute the deterministic FCNF model, setting the level of protection obtained in the second phase and simulating the realization of the uncertain parameter $O H_{i v}$ according to its historical data. Such process is repeated 100 times and we count 
how many times the problem becomes infeasible. Usually, solutions become infeasible due to higher simulated time spent at the port and a lack of protection. As $\Gamma=0$ leads to a deterministic solution and $\Gamma=K$ leads to a very conservative solution, we expect that the probability of infeasibility would be higher for the lower levels of robustness and it would diminish as $\Gamma$ increases.

The methodology we have presented enables one to determine the number of vessels needed to transport all the crude oil produced during a predefined planning horizon according to its own level of conservativeness and expected transportation costs. It also evaluates the trade-off between expected transportation costs for each level of robustness and the chance of that scenario remaining feasible when facing uncertainty in the time spent by vessels at ports. The probabilistic distribution of the time spent at ports (parameter $\mathrm{OH}_{i v}$ ) was defined according to historical data. Despite the parameter being indexed by each port $i$ and each vessel $v$, we consider that the time spent at port $O H_{i v}$ depends only on port $i$. We highlight that we observed no statistical dependence between the vessel and the port. In the next section, we present the computation experiments performed.

\section{Computational experiments}

We refer to the aforementioned case study to describe the instance of the MIR problem that we consider. This instance represents the geographic south and part of the southeast region of Brazil (see Figure 2). In this subsystem, there are 2 onshore terminals (A and B that supply one refinery each) and the crude oil production comes from 4 different FPSOs located on the southeast coast of Brazil. We allocated a potential fleet of 5 vessels of different sizes to supply these refineries during a planning horizon of 15 days. Numerical data are available at sal.aalto.fi/fabricio.

The uncertain parameter (time spent by vessels at each port) varies according to many reasons described in Section 2.2. We analyzed a two-year historical data set to estimate the mean time spent at ports $\left(O H_{i v}\right)$ and the maximum deviation from $O H_{i v}\left(O H D_{i v}\right)$. Since operations at FPSOs are more scattered than at onshore terminals, we analyzed the time spent at FPSOs as if they were only one and the time spent at each onshore terminal individually. Table 1 presents a statistical summary of the time spent at ports (duration) based on two-year historical data. 


\begin{tabular}{lccc}
\hline \multicolumn{1}{c}{ Statistic parameters } & Onshore terminal A & Onshore terminal B & FPSOs \\
\cline { 3 - 4 } Number of operations & 187 & 240 & 373 \\
Minimum duration (days) & 0.7 & 0.5 & 0.5 \\
Maximum duration (days) & 11.7 & 14.7 & 7.5 \\
Median (days) & 1.9 & 1.0 & 0.9 \\
Standar deviation (days) & 2.0 & 2.1 & 0.9 \\
\hline
\end{tabular}

Table 1: Two-year historic data time spent at ports.

We used the median value of the sample as the nominal value of the parameter time spent at port $\left(\mathrm{OH}_{i v}\right)$. Since time in the model is represented by discrete time periods, we approximate the value to an integer number of time periods. We estimate the maximum allowed deviation $\left(O H D_{i v}\right)$ to admit at least $80 \%$ of the operations of the historical data base. Table 2 presents these parameters and the comprehensiveness of the maximum admitted time spent at port $\left(O H_{i v}+O H D_{i v}\right)$ over the total number of occurrences. For instance, at onshore terminal $\mathrm{A}$, the maximum admitted time spent at port $\left(\mathrm{OH}_{i v}+\right.$ $O H D_{i v}$ ) represents $93 \%$ of the total amount of vessel operations on that terminal.

\begin{tabular}{|c|c|c|c|}
\hline Uncertain parameter & Onshore terminal A & Onshore terminal B & FPSOs \\
\hline$\overline{\mathrm{OH}_{i v}}$ & 2 & 1 & 1 \\
\hline$O H D_{i v}$ & 4 & 3 & 0 \\
\hline$O H_{i v}+O H D_{i v}$ comprehensiveness & $94 \%$ & $93 \%$ & $80 \%$ \\
\hline
\end{tabular}

Table 2: Uncertain parameters and time spent at ports.

In the robust optimization approach, one has to define the value of $\Gamma_{i}$. The parameter that defines the level of robustness $\left(\Gamma_{i}\right)$ is indexed by ports $i$. However, due to the minimal variation in vessel time spent at FPSOs (see Table 2), we consider no deviation in operations at FPSOs. Therefore, one must define the value of $\Gamma$ only for both onshore terminals ( $\mathrm{A}$ and $\mathrm{B})$. The value of $\Gamma_{i}$ may be interpreted as the maximum number of visits at port $i$ in which the time spent by the vessel may deviate from its mean value. For example, $\Gamma_{A}=0$ indicates that there is no deviation from the mean value of the port time in any operation at that terminal. $\Gamma_{B}=3$ indicates that the time spent by a vessel at port will deviate from its nominal value to the maximum deviation of up to 3 visits at onshore terminal B. According to the consumption rates and initial inventory level of each onshore terminal and the loading capacity of the ships, we estimate the maximum number of visits at each port (the maximum $\Gamma_{i}$ ). The maximum $\Gamma_{i}$ must be sufficient to continuously supply the onshore terminals and not let the inventory levels violate the minimum limits. In the instance that we have considered, the estimations of the maximum values are $\Gamma_{A}=4$ and $\Gamma_{B}=3$. Since the uncertainty in the time spent at each port follows its own historical 
probabilistic distribution, we have to combine both $\Gamma_{i}$ (for A and B) to define each level of robustness. Thus, we have 20 levels of robustness. Next, we present results for each level of robustness.

\subsection{Improving and assessing robust solutions}

We implemented the model using AIMMS 3.13 and solved it using GUROBI 5.5. We used an Intel Core i7 CPU with 8.0 GB RAM. First, we executed the RO-FCNF model and limited the computational time to 3,600 seconds for each level of robustness.

In Figure 4, we can assess the evolution of the objective function value obtained by the RO-FCNF (blue dashed line) and by the matheuristic (green line) as we change the level of $\Gamma$ for ports (A, B). The chart is divided in 4 blocks in which each block represents a level of $\Gamma_{B}$ (first block $\Gamma_{B}=0$, second block $\Gamma_{B}=1$, third block $\Gamma_{B}=2$ and fourth block $\Gamma_{B}=3$ ). For each level of $\Gamma_{B}$, we vary the level of $\Gamma_{A}$ from 0 to 4 . We use this block scheme in most charts of this section to represent the variation in the level of robustness.

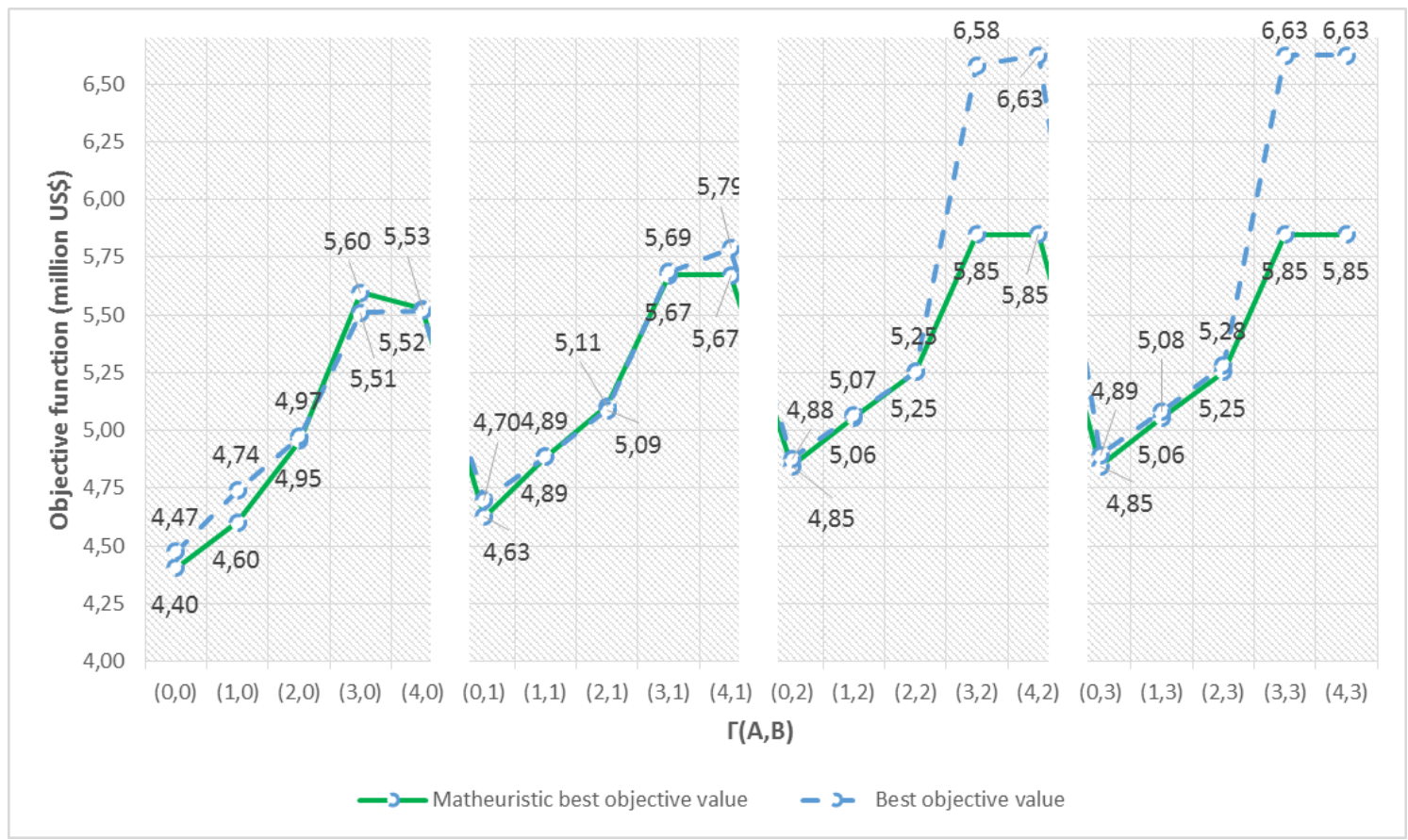

Figure 4: RO-FCNF using the conventional MILP solving.

Note that, for each block, the objective function value has a tendency of growth due to the increase in the robustness level. This behavior indicates that a more robust solution (ship scheduling) results in more expensive operational costs (objective function value).

The matheuristic approach was able to improve the objective function in most levels of 
robustness. On average, the objective function was improved by $2.8 \%$, and only in 3 levels of robustness was the objective function's value worse than using the conventional MIP. In addition to improving the objective function, the total computational time of the heuristic approach solution was reduced compared to the commercial MIP solver. Figure 5 presents the total computational time using the commercial MIP solver and the two phases of the matheuristic approach. The total reduction in computational time was approximately $33 \%$.

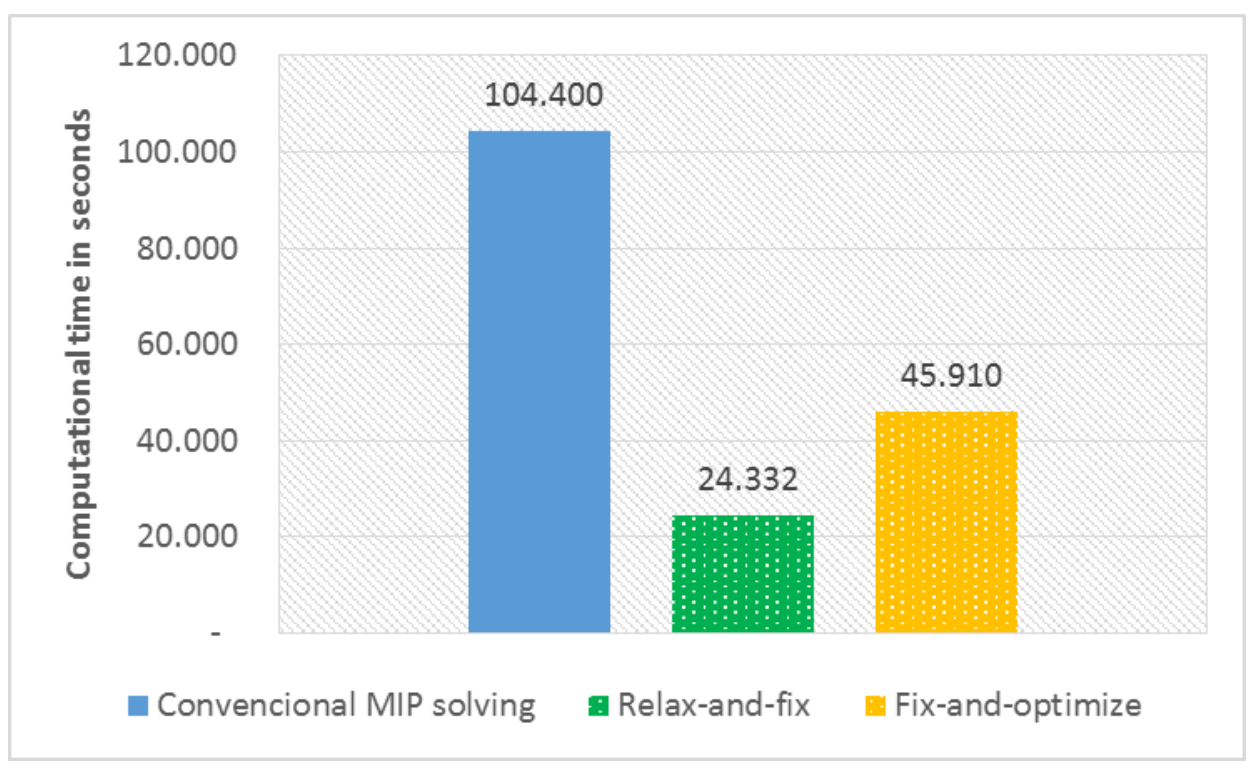

Figure 5: Computational times using the conventional MIP, the relax-and-fix and the fixand-optimize phases of the matheuristic approach.

Since the matheuristic approach provided better solutions for almost every level of robustness and reduced the total computational time, we considered the solutions obtained by the matheuristic approach as the reference for all of the analyses presented below.

As the level of robustness increases, the objective function value also increases. The level of robustness directly influences the how long vessels stay at ports. As it becomes higher, the time spent by vessels at port deviate from the nominal value in more visits. Consequently, it increases the demand for vessels, and it directly influences vessel availability.

In Figure 6, we present the relationship between the number of vessels (line) and the total vessel availability (bars), which represents the sum of vessel fleet availability. Note that as the level of robustness increases, the vessel availability also tends to increase. This is a way of protection against infeasibility since the model requires more vessels for a more 
robust solution.

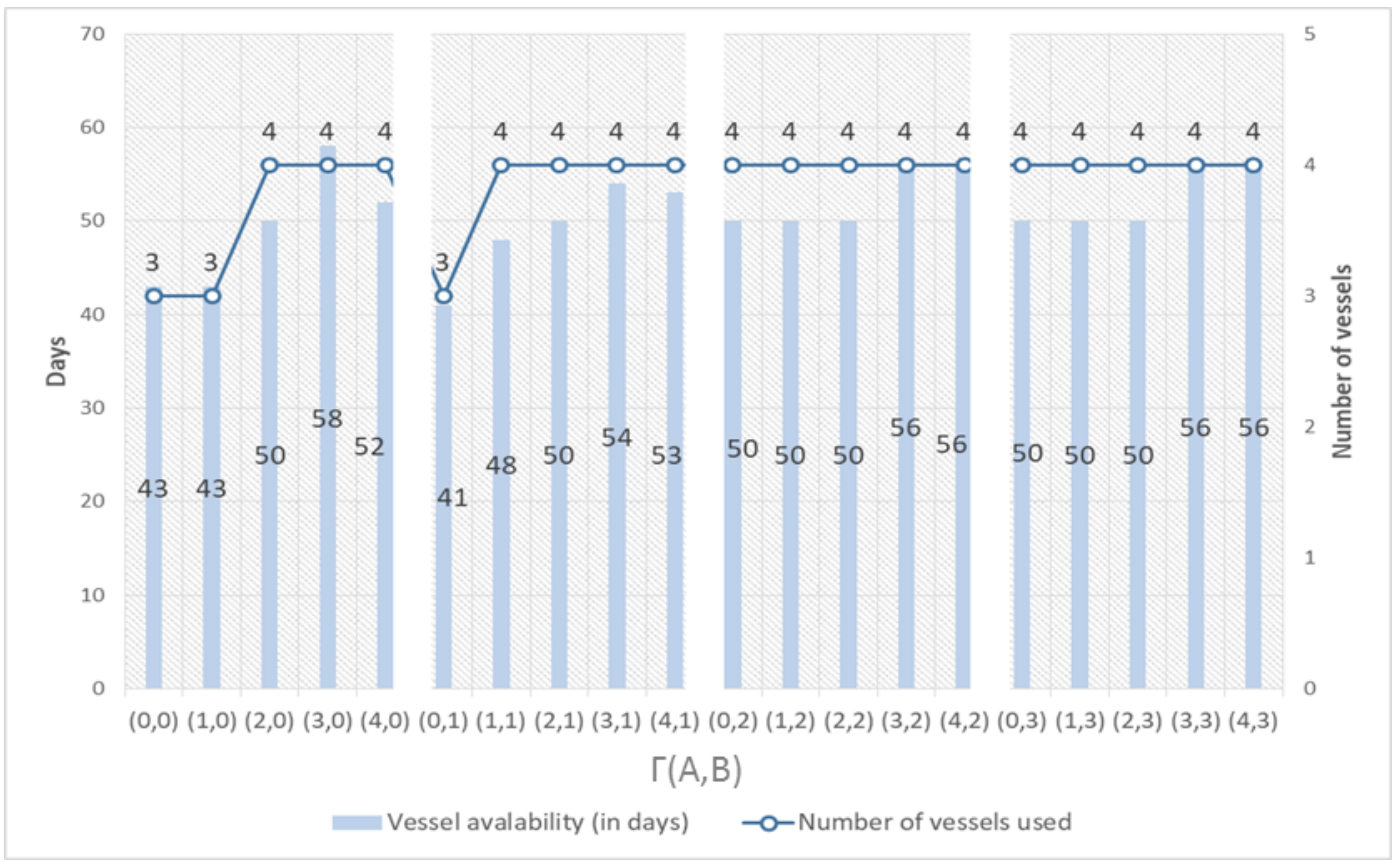

Figure 6: Number of vessels and total vessel availability.

Once it was observed how the model protects itself against infeasibility for different levels of robustness $(\Gamma)$, we proceeded to verify the probability of infeasibility of each solution on each level of robustness.

\subsection{Probability of infeasibility in robust methodology}

To verify the probability of infeasibility, we simulated 100 scenarios for the time spent at ports for each pair (port, vessel) according to the probability obtained by the historical data. The data are presented in Tables 1 and 2. For each scenario and each level of $\Gamma$, we executed the deterministic FNCF model by setting the fleet size and fleet availability defined for each level of $\Gamma$.

Figure 7 presents a comparison between the worst-case objective function (green continuous line) for each level of $\Gamma$, the average objective function (black dotted line) obtained from the simulation of the time spent at ports, and the probability of infeasibility of each level of protection given by the RO-FCNF model (red dashed line). It is important to mention that, during simulation phase, the execution of the deterministic FNCF model with fleet size and fleet availability fixed for each level of robustness provided optimal (or near optimal) results for every feasible solution obtained. We set a relative optimality gap of $3 \%$ as the stopping criterion for each execution. 
Note that the more robust the solution, the higher the chance of obtaining a feasible solution. We also observe the conservativeness of the robust solutions proposed by the RO-FCNF model.

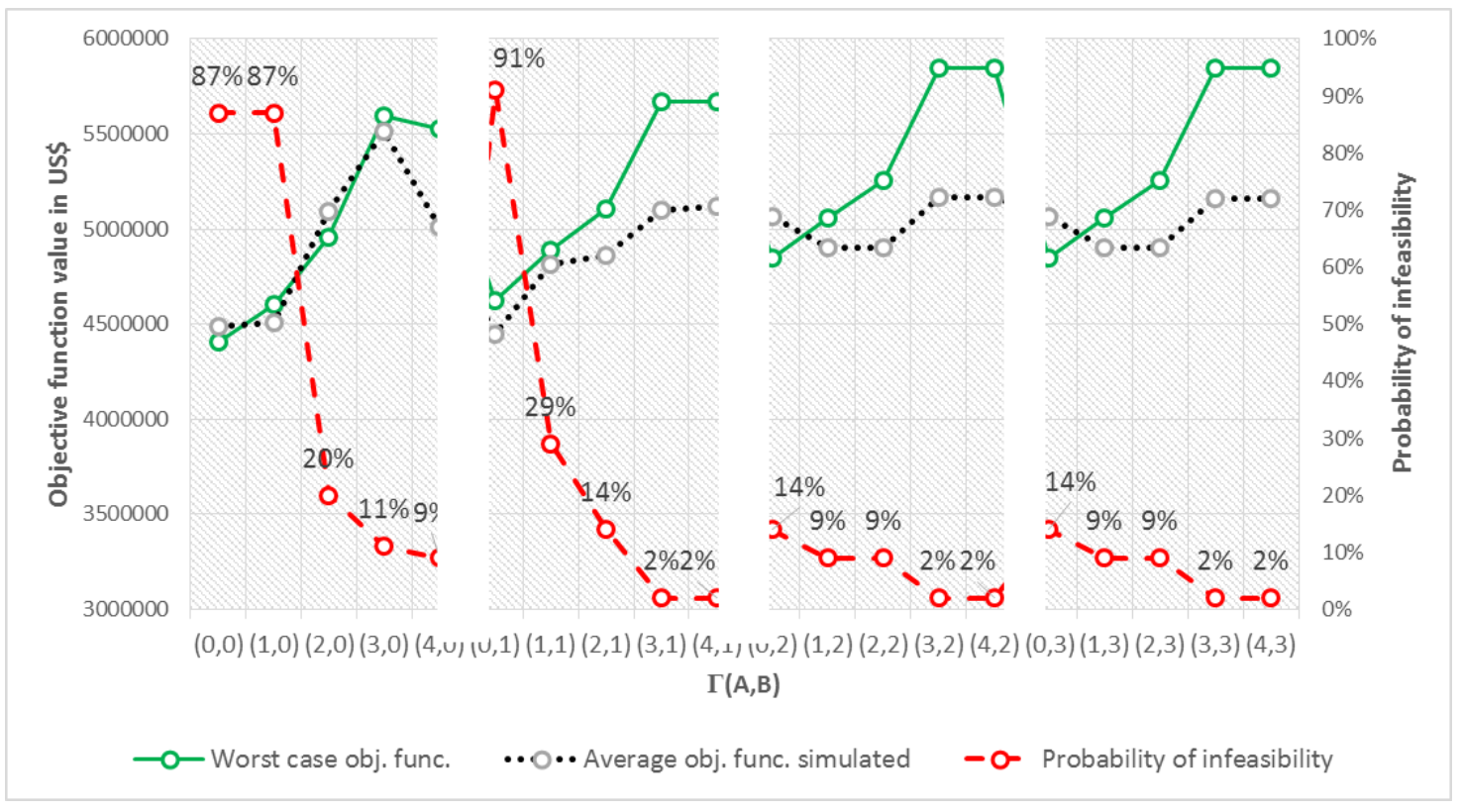

Figure 7: Evolution of the objective function of the worst case, average objective function simulated and the probability of infeasibility.

By examining Figure 7, one may decide how much risk one is willing to accept. The only previous decision is how many vessels one will allocate to each subsystem and when each vessel must be available for that subsystem (vessel availability). The simulation phase represents the daily scheduling decisions. In practice, it is made on a daily basis as uncertain parameters are revealed.

Note that for the nominal problem, where $\Gamma=(0,0)$ for $(\mathrm{A}, \mathrm{B}), 87 \%$ of the scenarios simulated are infeasible, although the objective function reaches the lowest value at around US\$ 4.4 million. This means that in $87 \%$ of the simulated scenarios, the fleet availability indicated in the solution of the nominal problem was not sufficient to carry the entire crude oil production from the production sites to the onshore terminals without letting inventory levels violate minimum and/or maximum limits (infeasible solutions). When one increases the level of robustness to $\Gamma=(3,1)$, the probability of infeasibility decreases to $2 \%$ and the increase in the average objective function is around US\$ 641 thousand, which corresponds to an approximately $13 \%$ increase in the value of the nominal problem's objective function. 
Regarding the trade-off decision between the risk of infeasibility and transportation costs, Figure 8 plots the efficient frontier for each level of robustness. The y-axis represents the average objective function simulated, while the $\mathrm{x}$-axis represents the probability of infeasibility and each dot represents a level of robustness. Analyzing the efficient frontier, one can identify the efficient solutions and the dominated solutions. The efficient solutions are those that for same probability of infeasibility have lower transportation costs, and dominated solutions are the ones that present higher transportation costs for the same probability of infeasibility.

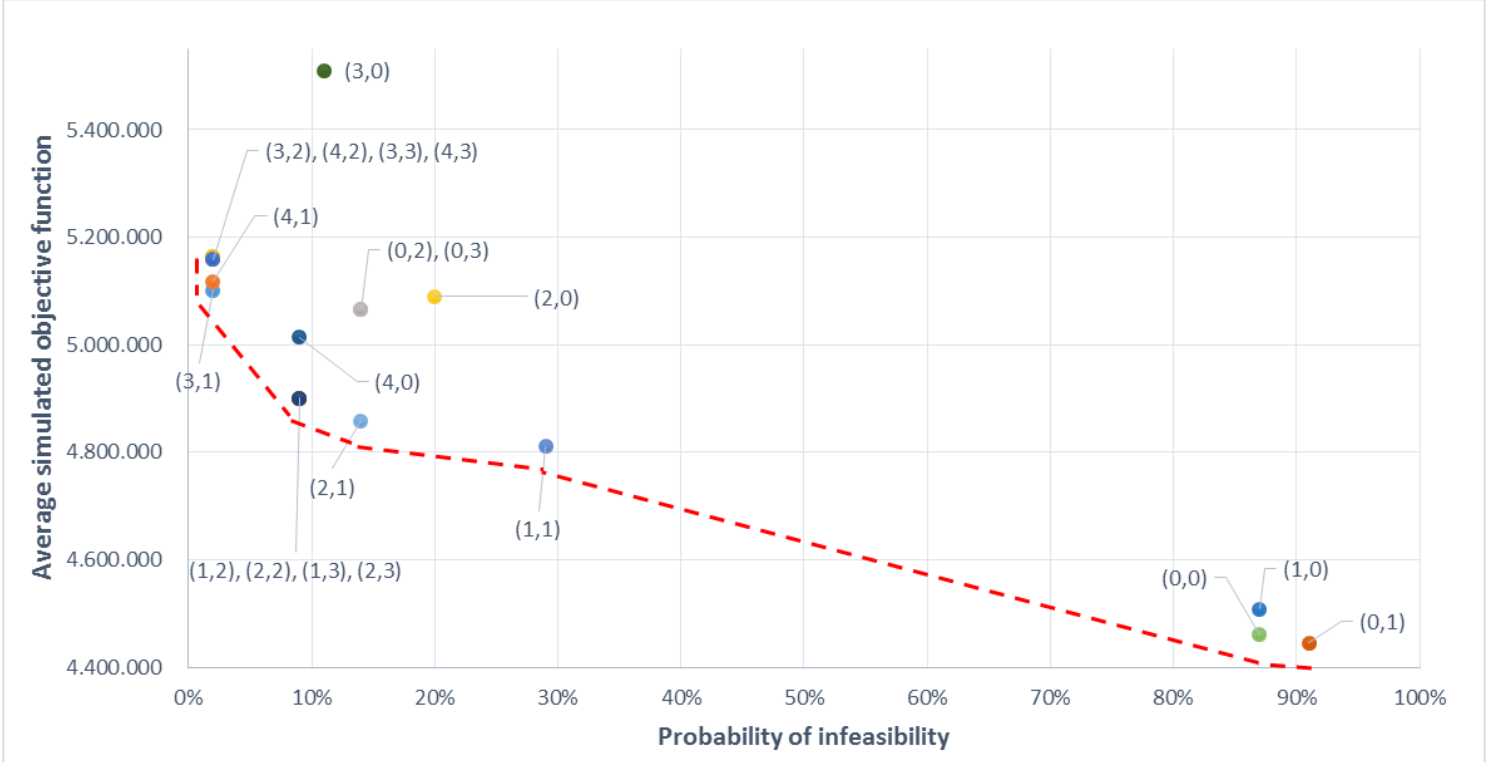

Figure 8: Efficiency frontier for levels of robustness.

By examining this chart, the decision maker may decide in advance which level of protection to give for a subsystem of a MIR problem by considering two important aspects (the probability of infeasibility and the increase in transportation costs (objective function)). This decision considers the uncertainty in the time spent at ports. In this example, if one considers approximately $10 \%$ of infeasibility probability to be allowable, then the levels of robustness $(2,1),(1,2),(2,2),(1,3)$ and $(2,3)$ are the ones that present the lower transportation costs for the level of conservatism. If one seeks a more robust solution that has a solution with a lower probability of infeasibility, then the level of robustness $(3,1)$ is the best option. This level is the one associated with the lowest average transportation costs for the lower probability of infeasibility. In practice, the solution considers the use of 4 vessels and 54 total time-periods of vessel availability. It also assumes that this level of protection is enough to accomplish $98 \%$ of the cases, considering the historical uncertainty of time spent at onshore terminals. 
An important contribution of such a robust methodology is supporting the decision maker in the ship scheduling activity. It gives in advance the risk of violating an inventory bound during the planning horizon for each level of robustness, and it assesses how much the level of allowed conservative would affect the operational costs. This trade-off analysis is fundamental for an efficient management of ship scheduling activity in a real-life MIR problem, such as the one being considered.

\section{Conclusion}

In this paper, we proposed a robust optimization approach for real-life MIR problems that considers uncertainty in the time spent by vessels at ports. This approach also quantifies the risk of infeasibility for different levels of robustness. Our experiments indicate that the use of the robust optimization approach to support ship-scheduling decisions considerably improves the quality of the decision. It means that by using the proposed approach, the decision maker can measure the risks of infeasibility (i.e., violating the inventory limits) and the impact of transportation costs for several scenarios with different robust levels. Therefore, one may decide within a range of options which solution best represents the best trade-off between conservativeness and increase the operational costs.

Despite the results obtained, there is still much space for further developments in the study of MIR problems, especially if considering uncertainty. As a future work, we propose studying alternatives to reduce the optimality gaps obtained when using the robust approach by means of improved solutions methods that may be based on decomposition methods and distributed computing. Another opportunity for future research is the inclusion of new uncertain parameters in the model, such as the production and consumption rates and vessel speed. 


\section{References}

AGRA, A.; ANDERSSON, H.; CHRISTIANSEN, M.; WOSLEY, L. A maritime inventory routing problem: Discrete time formulations and valid inequalities. Networks, v. 62, n. 4, p. 294-314, 2013.

AGRA, A.; CHRISTIANSEN, M.; DELGADO, A.; HVATTUM, L. M. A maritime inventory routing problem with stochastic sailing and port times. Computers \& Operations Research, v. 61, p. 18-30, 2015.

AGRA, A.; CHRISTIANSEN, M.; HVATTUM, L. M.; RODRIGUES, A MIP based local search heuristic for a stochastic maritime inventory routing problem. International Conference on Computational Logistics. Springer, Cham, p. 18-34, 2016.

AGRA, A.; CHRISTIANSEN, M.; HVATTUM, L. M.; RODRIGUES, F. Robust Optimization for a Maritime Inventory Routing Problem. Transportation Science, v. 52, n. 3, p. 509-525, 2018.

ASSIS, L. S.; CAMPONOGARA, E. A MILP model for planning the trips of dynamic positioned tankers with variable travel time, Transportation Research Part E, v. 96, p. 372-388, 2016

BEN-TAL, A., NEMIROVSKI, A. Robust solutions of linear programing problems contaminated with uncertain data. Mathematical Programming, v. 88, n. 3, p. 411424, 2000.

BERTSIMAS, D.; SIM, M. The price of robustness. Operations Research, v. 52, n. 1, p. 35-53, 2004.

CHRISTIANSEN, M. Decomposition of a combined inventory and time constrained ship routing problem. Transportation Science, v. 33, n. 1, p. 3-16, 1999.

CHRISTIANSEN, M.; FAGERHOLT, K.; RONEN D. Ship routing and scheduling: Status and perspectives. Transportation Science, v. 38, n. 1, p. 1-18, 2004. 
CHRISTIANSEN, M.; NYGREEN, B. Robust inventory ship routing by column generation. In: DESALNIERS, G.; DESROSIERS, J.; SOLOMON, M. M. (Eds.). Column Generation. Springer US, 2005. p. 197-224.

CHRISTIANSEN, M.; FAGERHOLT, K.; NYGREEN, B.; RONEN, D. Maritime transportation. Handbooks in Operations Research and Management Science, v. 14, p. 189-284, 2007.

CHRISTIANSEN, M.; FAGERHOLT, K. Maritime inventory routing problems. In: FLOUDAS, C. A.; PARDALOS, P. M. (Eds.). Encyclopedia of Optimization. Springer US, 2009. p. 1947-1955.

CHRISTIANSEN, M.; FAGERHOLT, K.; FLATBERG, T.; HAUGEN, O.; KLOSTER, O.; LUND, E.H. Maritime inventory routing with multiple products: A case study from the cement industry. European Journal of Operational Research, v. 208, n. 1, p. 86-94, 2011.

CHRISTIANSEN, M.; FAGERHOLT, K.; NYGREEN, B.; RONEN, D. Ship routing and scheduling in the new millennium. European Journal of Operational Research, v. 228, n. 3, p. 467-483, 2013.

DIZ, G; OLIVEIRA, F; HAMACHER, S. Improving maritime inventory routing: application to a Brazilian petroleum case. Maritime Policy and Management, v. 44, n. 1, p. 42-61, 2017.

DIZ, G. Maritime Inventory Routing: a practical assessment and a robust optimization approach. Doctoral Thesis, Pontifícia Universidade Católica, Brazil, Rio de Janeiro, 2017.

FLATBERG, T.; HAAVARDTUN, J.; KLOSTER, O.; LØKKETANGEN, A. Combining exact and heuristic methods for solving a Vessel Routing Problem with inventory constraints and time windows. Ricerca Operativa, v. 29, p. 55-68, 2000.

FURMAN, K. C.; SONG, J.-H.; KOCIS, G. R.; MCDONALD, M. K.; WARRICK, P. H. Feedstock routing in the ExxonMobil downstream sector. Interfaces, v. 41, n. 2, p. 149-163, 2011. 
GABREL, V., MURAT, C., THIELE, A. Recent Advances in Robust Optimization: An Overview. European Journal of Operational Research, v. 235, n. 3, p. 471-483, 2014.

GORISSEN, B. L., YANIKOGLU, I., HERTOG, D. A practical guide to robust optimization. Omega, v. 53, p. 124-137, 2015.

HALVORSEN-WEARE, E. E.; FAGERHOLT, K.; RÖNNQVIST, M. Vessel routing and scheduling under uncertainty in the liquefied natural gas business. Computers \& Industrial Engineering, v. 64, n. 1, p. 290-301, 2013.

PAPAGEORGIOU, D. J.; NEMHAUSER, G. L.; SOKOL, J.; CHEON, M.-S.; KEHA, A. B. MIRPLib - A library of maritime inventory routing problem instances: Survey, core model and benchmark results. European Journal of Operational Research, v. 235, p. 350-366, 2014.

ROCHA, R.; GROSSMANN, I. E.; DE ARAGÃO, M. V. S. P. Cascading knapsack inequalities: Reformulation of a crude oil distribution problem. Annals of Operations Research, v. 203, n. 1, p. 217-234, 2013.

SONG J.H.; FURMAN K. C. A maritime inventory routing problem: Practical approach. Computers \& Operations Research, v. 40, n. 3, p. 657-665, 2013.

SOYSTER, A. L. Convex programming with set-inclusive constraints and applications to inexact linear programming. Operations Research, v. 21, n. 5, p.1154-1157, 1973.

ZHANG, C.; NEMHAUSER, G.; SOKOL, J.; CHEON, MS.; PAPAGEORGIOU

D. Robust inventory routing with flexible time window allocation. Available on Optimization Online, 2015.

ZHANG, C.; NEMHAUSER, G.; SOKOL, J.; CHEON, MS.; KEHA A. Flexible solutions to maritime inventory routing problems with delivery time windows. Computers \& Operations Research, v. 89, p. 153-162, 2018. 


\section{Appendix 1}

Here we present a list of symbols used in the formulations in this paper. Sets and parameters are represented by capital letters and variables are in lower case. Some symbols may be represented by more than one letter for the sake of readability. The first part of symbols is used in the FCNF formulation.

Sets:

$N \quad$ set of all ports indexed by $i$ and $j$

Set of time periods indexed by $t$

$V \quad$ set of vessels indexed by $v$

NP $\quad$ set of loading ports indexed by $i$ and $j$

ND $\quad$ set of discharge ports indexed by $i$ and $j$

Parameters:

$C_{i j v}^{T} \quad$ sailing cost of vessel $v$ between ports $i$ and $j$

$C_{v}^{W} \quad$ waiting cost of vessel $v$ for each time period

$C_{i v}^{P} \quad$ port cost of port $i$ for vessel $v$

$O_{v} \quad$ the position of vessel $i$ in the beginning of planning horizon

$D E_{v} \quad$ the artificial end node for each vessel $v$

$T_{i j v} \quad$ sailing time of vessel $v$ between ports $i$ and $j$

$B_{i t} \quad$ number of berths available in port $i$ during time period $t$

$Q_{v} \quad$ the maximum amount of product to be loaded at one time period of ship $v$

$L 0_{v} \quad$ inventory on board of vessel $v$ in the beginning of the planning horizon

$K_{v} \quad$ vessel capacity

$D_{i t} \quad$ demand rate in the discharge port $i$ for each time period

$P_{i t} \quad$ production rate in the load port $i$ for each time period

$S M X_{i t} \quad$ upper bound of inventory level in port $i$ for each time period

$S M N_{i t} \quad$ lower bound of inventory level in port $i$ for each time period 
$S 0_{i} \quad$ inventory level at port $i$ in the beginning of the planning horizon

Variables:

$o_{i v t} \quad$ where $o_{i v t} \in\{0,1\}$

equal to 1 if the vessel is operating (loading/discharging) in port during time period $t$ and 0 otherwise,

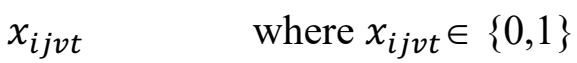

equal to 1 if vessel $v$ left port $i$ to port $j$ during time period $t$ and 0 otherwise,

$w_{\text {ivt }} \quad$ where $w_{\text {ivt }} \in\{0,1\}$

equal to 1 if vessel $v$ is waiting outside berth in port $i$ during time period $t$ and 0 otherwise,

$l_{v t} \quad$ where $l_{v t} \in \mathrm{R}$

inventory level on board vessel $v$ during time period $t$,

$q_{i v t \quad} \quad$ where $q_{i v t} \in \mathrm{R}$

quantity (un)load from/to vessel $v$ in port $i$ during time period $t$,

$s_{i t} \quad$ where $s_{i t} \in \mathrm{R}$

inventory level at port $i$ during time period $t$.

$o_{i v t}^{A} \quad$ where $o_{i v t}^{A} \in\{0,1\}$

indicates whether ship $v$ starts to operate at port $i$ in period $t$

$o_{i v t}^{B} \quad$ where $o_{i v t}^{B} \in\{0,1\}$

indicates whether ship $v$ continue to operate at port $i$ in period $t$

$f_{i j v t}^{X} \quad$ that indicates the load on board ship $v$ when traveling from port $i$ to port $j$, leaving port $i$ in period $t$,

$f_{i v t}^{O A} \quad$ that indicates the load on board ship $v$ when starting to operate at port $i$ in period $t$ and has not operated in period $t-1$,

$f_{i v t}^{O B} \quad$ that indicates the load on board ship $v$ before continuing to operate at port $i$ in period $t$ after having operated in time period $t-1$, 
$f_{i v t}^{W}$ that indicates the load on board ship $v$ while waiting during time period $t$ at port $i$.

The following symbols are used to reformulate FCNF model to become RO-FCNF model.

Sets

$K_{i v} \quad$ Set of coefficients $O H_{i v}$ that are subject to parameter uncertainty.

$U N B(v) \quad$ Subset of $\mathrm{T}$ that comprehend the time periods in the beginning of the planning horizon when each vessel is unavailable

UNE(v) Subset of $\mathrm{T}$ that comprehends the time periods at the end of planning horizon when each vessel is unavailable.

Parameters:

$T T$

$H R_{v}$

$O H_{i v}$

$O H D_{i v}$

$\widetilde{O H}_{i v}$

$\Gamma_{i v}$

$n h_{v}^{N T}$

$k_{i j v t}$

$\pi_{i v}, \rho_{i j v t}$
The last time period of the planning horizon $\mathrm{T}$,

The hire fee paid in a daily basis to vessels owner,

Nominal time spent by vessel $v$ at port $i$,

Maximum deviation observed for the time spent by vessel $v$ at port $i$,

Random variable representing the time spent by vessel $v$ at port $i$,

Represents the maximum number of coefficients parameters of constraints $(i, v)$ that can deviate from their nominal value. It adjusts the robustness of the decision. The parameter $\Gamma_{i v} \in\left[0,\left|K_{i v}\right|\right]$. Hence, the parameter $\Gamma_{i v}$ protects against deviations in up to $\left\lfloor\Gamma_{i v}\right\rfloor$ of these coefficients. In other words, we stipulate that nature will be restricted in its behavior, in that only a subset of the coefficients will change in order to adversely affect the solution.

Binary variable that indicates whether a vessel stayed idle during the entire planning horizon or not.

Auxiliary variable representing the scaled deviation of time spent at port $k_{i j v t}=\left(\widetilde{O H}_{i v}-O H_{i v}\right) / O H D_{i v}$,

Dual variables 


\section{Appendix 2}

Next, we present FCNF model, first proposed by Agra et al. (2013).

Objective function:

$\min \sum_{v \in V} \sum_{i \in N\left\{O_{v}\right\}} \sum_{j \in N \cup\left\{D E_{v}\right\}} \sum_{t \in T} C_{i j v}^{T} \cdot x_{i j v t}+\sum_{v \in V} \sum_{i \in N} \sum_{t \in T} C_{i v}^{P} \cdot x_{i j v t}+$

$$
\sum_{v \in V} \sum_{i \in N} \sum_{t \in T} C_{v}^{W} \cdot w_{i v t}
$$

The minimization function (1) contains transportation costs, operation costs and waiting costs.

Constraints

$\sum_{j \in N \cup\left\{D E_{v}\right\}} \sum_{t \in T} x_{O_{v} j v t}=1, \forall v \in V$,

$\sum_{i \in N \cup\left\{O_{v}\right\}} \sum_{t \in T} x_{i D E_{v} v t}=1, \forall v \in V$

$o_{i v, t-1} \leq \sum_{j \in N \cup\left\{D E_{v}\right\}} x_{i j v t}+o_{i v t}, \forall v \in V, i \in N, t \in T$,

$o_{i v, t-1} \geq \sum_{j \in N \cup\left\{D E_{v}\right\}} x_{i j v t}, \quad \forall v \in V, i \in N, t \in T$,

Constraints (2) and (3) guarantee that every ship leaves from its artificial origin port and finishes the voyage at its artificial destination port. Constraints (4) determine that after an operation period, a vessel can continue to operate or sail the port in the next period and constraints (5) ensure that before leaving a port, the vessel must have operated there in the time period immediately before.

$$
\begin{aligned}
& \sum_{j \in N \cup\left\{o_{v}\right\}} x_{j i v, t-T_{j i v}}+w_{i v, t-1}=w_{i v t}+o_{i v t}^{A}, \forall v \in V, i \in N, t \in T, \\
& o_{i v t-1}^{A}+o_{i v t-1}^{B}=o_{i v t}^{B}+\sum_{j \in N \cup\left\{D E_{v}\right\}} x_{i j v t}, \forall v \in V, i \in N, t \in T, \\
& o_{i v t}^{A}+o_{i v t}^{B}=o_{i v t}, \quad \forall v \in V, i \in N, t \in T,
\end{aligned}
$$

$\sum_{v \in V} o_{i v t} \leq B_{i t}, \forall i \in N, t \in T$

Constraints (6) and (7) represent vessel's movement along the time-space network structure. At every time period, the vessel must be leaving a port, waiting to operate or operating at a port. These constraints also connect the time when a vessel leaves one port $j$ to the time when the same vessel arrives at a port $i$. Constraints (8) provide the 
coordination between the path of the ships and the loading or discharging of crude oil in a port. Constraints (9) give berth restriction at each node and consequently waiting time.

$0 \leq q_{i v t} \leq Q_{v} o_{i v t}, \forall v \in V, i \in N, t \in T$

$\sum_{j \in N \cup\left\{o_{v}\right\}} f_{j i v, t-T}^{X}+f_{i v, t-1}^{W}=f_{i v t}^{W}+f_{i v t}^{O A}, \forall v \in V, i \in N, t \in T$,

$f_{i v, t-1}^{O A}+f_{i v, t-1}^{O B}+q_{i v, t-1}=f_{i v t}^{O B}+\sum_{j \in N \cup\left\{D E_{v}\right\}} f_{i j v t}^{X}$,

$\forall v \in V, i \in N P \cup\left\{O_{v}\right\}, t \in T$

$f_{i v, t-1}^{O A}+f_{i v, t-1}^{O B}-q_{i v, t-1}=f_{i v t}^{O B}+\sum_{j \in N \cup\left\{D E_{v}\right\}} f_{i j v t}^{X}$,

$\forall v \in V, i \in N D \cup\left\{O_{v}\right\}, t \in T$,

$f_{O_{v} j v t}^{X}=L 0_{v} \cdot x_{O_{v} j v t}, \quad \forall v \in V, j \in N \cup\left\{D E_{v}\right\}, \quad t \in T$

The quantity to be (un)loaded $\left(q_{i v t}\right)$ must be less than the maximum amount of product to be loaded or discharged from ship v (constraints 10). The load onboard the vessel on each arc of the network flow is controlled in constraints (11)-(131). Initial inventory level onboard the vessel is given in constraints (14).

$0 \leq f_{i j v t}^{X} \leq K_{v} \cdot x_{i j v t}, \forall v \in V, i \in N \cup\left\{O_{v}\right\}, j \in N \cup\left\{D E_{v}\right\}, t \in T$

$0 \leq f_{i v t}^{O A} \leq K_{v} . o_{i v t}^{A}, \quad \forall v \in V, i \in N, t \in T$,

$0 \leq f_{i v t}^{O B} \leq K_{v} \cdot o_{i v t}^{B}, \forall v \in V, i \in N, \quad t \in T$,

$0 \leq f_{i v t}^{W} \leq K_{v} \cdot w_{i v t}, \forall v \in V, i \in N, t \in T$

The variables upper bounds (vessel capacity) and non-negativity constraints are expressed in $(15)-(18)$ :

$s_{i, t-1}+\sum_{v \in V} q_{i v t}=D_{i t}+s_{i t}, \forall i \in N D, t \in T$,

$s_{i, t-1}+P_{i t}=\sum_{v \in V} q_{i v t}+s_{i t}, \forall i \in N P, t \in T$,

$S M N_{i t} \leq s_{i t} \leq S M X_{i t}, \forall i \in N, t \in T$

$s_{i 0}=S 0_{i}, \forall i \in N$,

The inventory level at each port is controlled during every time period of the planning 
horizon at load and discharge ports (constraints 19 and 20). Constraints (21) give the operational range for inventory levels and constraints (22) define initial inventory levels at each port.

\section{Reformulations proposed}

The first reformulation that we propose represents the fixed costs of the fleet. To achieve that, we add hiring costs to the objective function and formulate constraints that ensure whether the vessel is used during that planning horizon.

Objective function

$$
\begin{aligned}
& \min \sum_{v \in V} \sum_{i \in N \cup\left\{O_{v}\right\}} \sum_{j \in N \cup\left\{D E_{v}\right\}} \sum_{t \in T} C_{i j v}^{T} \cdot x_{i j v t}+\sum_{v \in V} \sum_{i \in N} \sum_{t \in T} C_{i v}^{P} x_{i j v t}+ \\
& \sum_{v \in V} \sum_{i \in N} \sum_{t \in T} C_{v}^{W} w_{i v t}+\sum_{v \in V}\left(\mathbf{1}-\boldsymbol{n} \boldsymbol{h}_{v}^{N \boldsymbol{T}}\right) \boldsymbol{H} \boldsymbol{R}_{\boldsymbol{v}} \boldsymbol{T} \boldsymbol{T}
\end{aligned}
$$

Constraints

$n h_{v}^{N T}=\sum_{i=O_{v}} \sum_{j=D E_{v}} \sum_{t \in T} x_{i j v t} \quad \forall v \in V$

In (23), we add (in bold) the fixed costs for every vessel that is utilized during the planning horizon to the original objective function. Note that regardless of whether the vessel is utilized for one day or the entire planning horizon, the hire fee is charged for the complete planning horizon. Constraints (24) ensure that if a vessel goes directly from the artificial origin node to the artificial destination node, then it stays idle for the entire planning horizon.

$\sum_{t \in T} o_{i v t} \geq \sum_{t \in T, j \in N} O H_{i v} \cdot x_{i j v t}, \quad \forall i \in N, v \in V$,

Constraints (25) determine that the number of time-periods a vessel $v$ spends at port $i$ must be at least the product of the nominal time spent at port $O H_{i, v}$ and the number of visits of vessel $v$ at port $i$. For example, if the nominal time spent at port $i$ is equal to 1 day and vessel $v$ visits the port 3 times, it means that the sum of operation time $\sum_{t \in T} o_{i v t}$ (or time spent at port) of the vessel $v$ at port $i$ during the entire planning horizon must be equal to or higher than 3 (the product of the nominal time spent at port $i$ and the number of visits of vessel $v$ at port $i$ ). 


\section{Appendix 3}

To consider uncertainty in the time spent at ports (parameter $O H_{i v}$ ), we reformulate constraints (25) into a stochastic version (25s), where $O H_{i v}$ may deviate up to its maximum deviation $O H D_{i v}$ according to its scalar deviation $k_{i j v t}$ :

$\sum_{t \in T} o_{i v t} \geq \sum_{t \in T, j \in N} O H_{i, v} \cdot x_{i j v t}+\sum_{t \in T, j \in N} O H D_{i v} \cdot x_{i j v t} \cdot k_{i j v t}, \forall i \in N, v \in V$

In order to build the robust counterpart of the model, it is necessary to reformulate constraints (25s). First, we adopt the robust paradigm, which considers that uncertainty will behave as worse as possible. In other words, $(25 \mathrm{~s})$ indicates that the solution would be feasible for every realization of the time spent at port.

$\Phi(x, \Gamma)=\operatorname{Max}_{k}\left\{\sum_{t \in T, j \in N} O H D_{i v} \cdot x_{i j v t} \cdot k_{i j v t} \mid \sum_{t \in T, j \in N} k_{i j v t} \leq \Gamma_{i v}, k_{i j v t} \geq 0\right\}$

Note that (26) protects against the uncertainty for every visit of a vessel $v$ at a port $i$ and it is known as protection function. We disregard $k_{i j v t} \in[-1,0]$, because we intend to protect only against the worst cases. Moreover, the parameter $\Gamma_{i v}$, introduced to adjust the model robustness against the conservatism of the solution, can be understood as the maximum number of the uncertain parameters that can deviate from their nominal values. $\Gamma_{i v}$ may take values in the interval $[0,|K|]$, where $|K|$ represents the maximum number of visits a port $i$ may receive during the entire planning horizon.

The robust counterpart of $(25)$ is

$\sum_{t \in T} o_{i v t} \geq \sum_{t \in T, j \in N} O H_{i, v} \cdot x_{i j v t}+\Phi(x, \Gamma), \forall i \in N, v \in V$

Applying the robust optimization technique developed by Bertsimas and Sim (2004), we formulate the auxiliary problem (28)-(30). Its objective is to maximize the sum of all deviations over the set of all admissible realizations of the uncertain parameters.

$\operatorname{Max}_{k} \sum_{t \in T, j \in N} O H D_{i v} \cdot x_{i j v t}^{*} \cdot k_{i j v t}$

Subject to:

$\sum_{j, t} k_{i j v t} \leq \Gamma_{i v}, \forall i, v$

$0 \leq k_{i j v t} \leq 1, \forall i, j, v, t$

If $\Gamma_{i v}=0, k_{i j v t}$, for all $i, j, v, t$, is forced to be 0 , so that the random variable $\widetilde{O H}_{i v}$ is equal 
to their mean value $\mathrm{OH}_{i, v}$ and there is no protection against uncertainty. On the other hand, when $\Gamma_{i v}=|K|, k_{i j v t}$, for all $i, j, v, t$, is forced to be 1 (in this particular problem) and constraints $(25 \mathrm{~s})$ is completely protected against uncertainty, yielding a very conservative solution. For values between 0 and $|K|$, the decision-maker can tradeoff between the protection level and the degree of conservatism of the solution.

Following the same rationale of Bertsimas and Sim (2004), the dual of the model (28)(30) is stated as follows:

$\operatorname{Min}_{\pi, \rho} \pi_{i v} \cdot \Gamma_{i v}+\sum_{t \in T, j \in N} \rho_{i j v t} \quad, \forall i, v$

Subject to:

$\pi_{i v}+\rho_{i j v t} \geq O H D_{i v} \cdot x_{i j v t}, \forall i, j, v, t$

$\rho_{i j v t} \geq 0, \forall i, j, v, t$

$\pi_{i v} \geq 0 \quad, \forall i, v$

This dual problem has two dual variables $\left(\pi_{i v}, \rho_{i j v t}\right)$ that are associated to constraints (28) and (30), respectively. By strong duality, as model (28)-(30) is feasible and bounded for all $\Gamma_{i v} \in\left[0,\left|K_{i v}\right|\right]$, then the dual problem (31)-(34) is also feasible and their objective function values coincide.

Substituting (31)-(34) in constraints (27), the following robust linear set of constraints are obtained.

$\sum_{t \in T, j \in N} O H_{i v} \cdot x_{i j v t}+\pi_{i v} \cdot \Gamma_{i v}+\sum_{t \in T, j \in N} \rho_{i j v t}-\sum_{t \in T} o_{i v t} \leq 0, \forall i \in N, v \in V$,

$\pi_{i v}+\rho_{i j v t} \geq O H D_{i v} \cdot x_{i j v t} \quad, \forall i, j, v, t$

$\rho_{i j v t} \geq 0, \forall i, j, v, t$

$\pi_{i v} \geq 0, \forall i, v$

Adding (35)-(38) to the original FCNF formulation, we have now the RO-FCNF formulation. This model minimizes transportation costs and ensures that up to $\Gamma$ coefficients deviate their value from the mean time spent at a port within the permitted interval $\left(\left[O H_{i v}-O H D_{i v}, O H_{i v}+O H D_{i v}\right]\right)$, then the solution of the robust optimization model will remain feasible. In other words, the solution of this model is a robust solution. 


\section{Appendix 4}

Next, we present additional constraints in the deterministic FCNF model used to set vessel availability for each level of $\Gamma$.

$\sum_{t \in U N B_{v}} x_{i j v t}=0 \quad \forall i, j \in N, \forall v \in V$

$\sum_{t \in U N E_{v}} x_{i j v t}=0 \quad \forall i, j \in N, \forall v \in V$

Constraints (39) and (40) define the period of vessel unavailability in the beginning and at the end of the planning horizon, respectively. It is important to highlight that constraint (39)-(40) are only used in the simulation phase of the robust optimization approach, because the unavailability periods (in the beginning and at the end of planning horizon) are only known after the robust model is solved for each level of $\Gamma$ during the second phase of the methodology. 